\title{
Clinical Applications of Age-Related Macular Degeneration Genetics
}

\author{
John Paul SanGiovanni and Emily Y. Chew \\ National Eye Institute, Clinical Trials Branch, Division of Epidemiology and Clinical Applications, \\ National Eye Institute, National Institutes of Health, Bethesda, Maryland 20892 \\ Correspondence: echew@nei.nih.gov; jpsangio@post.harvard.edu
}

\begin{abstract}
Understanding genetic causes of age-related macular degeneration (AMD) will eventually yield effective discoveries and improvements in predictive/prognostic methods. These include, but are not limited to, reliable disease prediction (screening for increased discrimination of clinical risk), differential classification of AMD subtypes with biomarkers (development of risk-linked molecular taxonomies), selection of optimal preventive and therapeutic interventions (guided by a biologically meaningful understanding of treatment response), and drug dosing. In this review, we discuss clinical applications informed by key findings in AMD genetics, and provide commentary on leveraging extant and forthcoming evidence to improve AMD risk prediction, AMD classification, and knowledge on the genetic basis of drug activity and toxicity. Advances in translating AMD genetics findings for AMD risk prediction require development of a genetics-based causality for AMD incidence and progression. Molecular subtyping of AMD phenotypes requires a set of dynamic biomarkers presenting prognostic value; although these have yet to be identified, the formation of multidisciplinary teams and their participation in large-scale consortia may yield promising results. Drugs targeting complement and vascular endothelial growth factor (VEGF) systems are under evaluation, and forthcoming work on rare variants and noncoding DNA in AMD pathogenesis will likely reveal biochemical pathways enriched with AMD-associated genetic variants. Pharmacologic targets in these pathways may inform a rational and effective therapeutic approach to preventing and treating this sight-threatening disease.
\end{abstract}

ge-related macular degeneration (AMD) is
a complex sight-threatening disease of pub-
lic health significance (Miller 2013). Knowledge
on the nature of AMD phenotypes and related
pathogenic processes (as well as on constraints
in approaches to investigation) has influenced
our ability to make inferences on a genetics-
based causality of this disease. Such inferences
are necessary in the development of feasible clin-
ic-based approaches for effective AMD preven- tion, prognosis, and treatment (Gorin 2012). In this review we discuss clinical applications informed by key findings in AMD genetics, and provide commentary on leveraging extant and forthcoming evidence to improve AMD risk prediction, classification, and knowledge on the genetic basis of drug activity and toxicity. We start with a definition of AMD phenotypes and follow with an overview of what research in AMD genetics has told us about the nature and

Editors: Eric A. Pierce, Richard H. Masland, and Joan W. Miller

Additional Perspectives on Retinal Disorders: Genetic Approaches to Diagnosis and Treatment available at www.perspectivesinmedicine.org

Copyright (C) 2014 Cold Spring Harbor Laboratory Press; all rights reserved; doi: 101101/cshperspect.a017228

Cite this article as Cold Spring Harb Perspect Med 2014;4:a017228 
pathogenesis of the disease. These sections are followed by examples of clinical applications in AMD, informed by the aggregate of genetic findings from allied fields of molecular biology, biochemistry, biophysics, cell biology, and bioinformatics. We conclude with commentary on the opportunity for translating existing and emerging results for the purpose of improving clinical care.

\section{AMD PHENOTYPES}

Features of advanced, late stages of sight-threatening AMD include pathologic neurodegenerative and/or vasoproliferative changes to the interfaces between the photoreceptors, retinal pigment epithelium (RPE), and choroid vascular network. Neurodegenerative AMD (geographic atrophy) is characterized by one or more notable regions ( $\geq 175 \mu \mathrm{m})$ of RPE depigmentation or loss, with concomitant degeneration in the choriocapillaris. Atrophic AMD subtypes are represented by abnormal focal, banded, patchy, or diffuse patterns on fundus autofluorescence measurements (Bindewald et al. 2005). Neovascular AMD involves abnormal development of nascent vessels projecting from the choriocapillaris to the subretinal (Type 1) and/ or sub-RPE space (Type 2). Polypoidal choroidal vasculopathy is a subtype of neovascular AMD with clusters of spheroid, choroid-derived vessels typically settling in the sub-RPE space. Retinal angiomatous proliferation is also a discrete neovascular AMD subtype; in this condition, intraretinal neovascular lesions connect with vessels from the choroidal circulations. The Beckman Initiative for Macular Research has published a phenotypic classification of AMD based on consensus of 30 opinion leaders in the field (Ferris et al. 2013). A current goal of AMD genetics is to identify biomarkers that can be used to distinguish these heterogeneous clinical subtypes (Gorin 2012).

\section{STATE OF SCIENCE IN AMD GENETICS}

Observations on familial associations in AMD were published by Gass nearly four decades ago (reviewed in Miller 2013), and broadly project- ed the concept of a hereditary component to the disease. Relative risk of developing AMD among first-degree relatives is 6-12 times higher than in the general population (Klaver et al. 1998), and findings from family-based studies on sibling recurrence risk and disease coincidence in twins suggest that the proportion of variability in AMD risk attributed to heritability ranges from $45 \%$ to $70 \%$ (Seddon et al. 2005). Details on the large body of results from familial aggregation, segregation, linkage, and twin study approaches are presented by Haddad et al. (2006) and Lim et al. (2012). The key message is that hereditary forms of AMD have shown linkage peaks on at least 17 chromosomes (Gorin 2012); in at least 15 instances, these loci contain common AMD-associated DNA sequence variants present in sporadic (nonfamilial) cases of the disease (Gorin 2012; Fritsche et al. 2013).

Disease-gene relationships in sporadic cases of AMD have been investigated most commonly using the genome-wide association (GWA) study approach paired with linkage disequilibrium mapping. This approach involves broad DNA scans on hundreds of thousands of common sequence variants in thousands of people for the purpose of mapping diseaserelated loci, and is predicated on the idea that differences between healthy and complex disease states may be partially manifested by multiple common DNA sequence variants - a concept known as the "common disease-common variants hypothesis" (Lander 1996; Manolio et al. 2009). In 2005, the value of the GWA design for efficiently and reliably identifying diseaseassociated loci (from common sequence variants) was shown in the first set of reports implicating the complement factor $\mathrm{H}(\mathrm{CFH})$ $\mathrm{Y} 402 \mathrm{H}$ variant (chromosome 1q31 locus) in advanced AMD (Klein et al. 2005). These findings, and those describing common variants within another prominent AMD-associated linkage peak on chromosome 10q26, have been replicated in numerous cohorts of subjects with American, Asian, or European ancestry (Haddad et al. 2006; Gorin 2012; Fritsche et al. 2013). In 2013, researchers from a multinational consortium reported results on 19 common susceptibility 
loci for AMD from analyses on nearly 80,000 people; in aggregate, these loci explain $10 \%-$ $30 \%$ of phenotypic variation in the disease (Fritsche et al. 2013).

The 19 AMD susceptibility loci were identified with the GWA study approach and have shown characteristics (presented below) of other large-scale efforts noted by Manolio et al. (2009) four years following publication of the initial AMD-Y402H findings. From this study and follow-up commentaries on the topic (Manolio and Green 2011; Manolio 2013), a key message emerges: The many robust relationships elucidated by the GWA approach have offered meaningful guidance on the underlying biology of complex diseases. Furthermore, by amplifying GWA-derived signals with a careful curation process (leading to a more complete annotation of noncoding DNA overlapping regulatory elements, rare/low frequency variants, and structural variants), one may develop meaningful clinical applications. To adequately inform a translation to the clinic, these efforts require extended work (beyond annotation) aimed to identify disease-associated rare, structural, and regulatory variants. Strengths and limitations to inference based on the method are (1) the GWA approach has been successful in identifying novel susceptibility loci for guiding efficient searches on plausible etiologic processes and strengthening insights on phenotypes (Cichon et al. 2009); (2) the magnitude of effects in GWA-based disease-gene associations have been modest (in the range of a 10\%-50\% change in disease likelihood for the presence of each risk allele) due to aspects of disease biology and chip design constraints-chips carry probes mainly for common single-nucleotide polymorphisms (SNPs), rather than rare SNPs or structural variants, which include copy number variants, inversions, deletions and insertions; (3) individual GWA studies are generally underpowered to detect modest effect sizes typically observed for common variants, which may lead to underestimates of the number of disease-gene relationships, whereas combining data from multiple studies has produced informative and independently replicated findings (Fritsche et al. 2013); (4) positional information yielded by GWA studies has almost never directly identified a causal variant (Gorin 2012); (5) the range of associated "functional" SNPs (those that lead to changes in processing or expression of the gene transcript or structural and subsequent functional integrity of the protein) coinherited with an AMD-associated SNP may extend $100 \mathrm{~kb}$, and these are candidate regions for fine mapping or resequencing, copy number variants, and promoter regions; and (6) a region may house multiple independent risk variants.

Disease-associated rare variants typically show larger effect sizes and higher predictive value than those of common variants; accordingly, a number of well-funded groups are now directing their efforts to searching for rare variants (McCarthy et al. 2008; Gorin 2012; Manolio et al. 2013). The Seddon group presented the first major finding on a rare and highly penetrant AMD-associated variant in the $C F H$ locus (Raychaudhuri et al. 2011). The complement component 3 (C3) gene was recently shown to contain a rare AMD-associated variant yielding a greater effect size than those attributed to common AMD-associated variants in the gene; these discoveries were made efficiently after results from GWA studies identified regions for deep sequencing to be used in a cohort large enough to attain stable estimates of effect (Seddon et al. 2013). Gorin offers detailed commentary on the importance of investigating rare variants in complex disease, acknowledging the possibility that genes encoding key etiologic factors may not carry common sequence variants (Gorin 2012).

The central message of this section is that we have been somewhat limited in moving AMD genetics to clinical applications by our dependence on findings from studies designed to interrogate common sequence variants, characterized mainly as SNPs. Imputation and analyses conducted to place these common SNPs into plausible biologic pathways implicated in AMD-related pathology may better guide us toward finding functional DNA sequence variants (that have posttranscriptional and posttranslational effects) in coding or noncoding genomic regions. There is greater appreciation for 
examining the role of rare SNPs, variation in noncoding DNA linked to regulatory elements (microRNA, histone accessibility marks, DNase hypersensitivity clusters, and transcription factor binding sites), and structural variants (copy number variants, inversions, deletions, and insertions) in AMD. Clearly, in developing effective clinical applications of AMD genetics, we must turn to allied fields of biochemistry, biophysics, and cell biology to integrate evidence on AMD-associated exposures, pathogenesis, and pathophysiology in human cohorts and model systems.

\section{KEY DISCOVERIES IN AMD GENETICS: PATHOGENESIS AND PATHOPHYSIOLOGY IN CONTEXT}

Recently published works on the state of genomic science in AMD research (Miller 2013) and use of genetics-based findings for inference on AMD causality, risk, and therapeutics (Gorin 2012) provide valuable overviews on systems of genes that encode proteins implicated in AMD pathogenesis and pathophysiology. These systems include the alternative complement pathway, the innate immune response pathway, the chromosome 10q26 locus, extracellular matrix/cell adhesion complex genes, lipid metabolism/transport/signaling-related genes, the mitochondrial genome, vitamin D pathway genes, angiogenesis-related genes, iron metabolism genes, and cellular stress and toxicity genes. Reviews over the past decade have also addressed concepts of pathogenic mechanisms in the context of the human genome (Ambati et al. 2003; Haddad et al. 2006; Hageman et al. 2008; Bird 2010). Initially, family-based and GWA studies applied an empirical (data-driven) approach to identify and characterize mechanisms involved in AMD pathogenesis; hypothesis-driven research is now the mainstay, guided by or supported with evidence from model systems, epidemiologic studies, and clinical observations. There is general consensus that key mechanisms in AMD pathogenesis involve dysregulation of processes leading to (1) abnormal angiogenesis; (2) age-related deposition of amyloid, lipids, or lipofuscin; (3) apoptosis; and
(4) activation or modulation of (a) cellular stress pathway constituents, (b) inflammatoryimmune response, or (c) vascular resistance. Lists of AMD-associated genes that drive these processes are provided in recent reviews (Haddad et al. 2006; Gorin 2012; Miller 2013). Pathway and network enrichment analyses are now entering the evidence base (SanGiovanni and Mehta 2009; Silveira et al. 2010; Newman et al. 2012; Fritsche et al. 2013; SanGiovanni and Lee 2013) and may offer meaningful guidance on novel therapies. The majority of targeted therapies for AMD have been directed at inhibiting pathologic angiogenesis through perturbation of VEGF signaling or to alter activity of complement-based systems.

\section{INTERPRETATION AND APPLICATION OF FINDINGS ON GENETICS-BASED CAUSALITY OF AMD}

Successful efforts to develop effective clinicbased preventive, prognostic, and therapeutic applications for AMD will require integration of knowledge on AMD-associated genes and their encoded proteins with evidence from genetically modified model systems and epidemiologic, clinical, biochemical studies (Gorin 2012). In this section we discuss aspects of genetic findings essential for designing effective clinical applications (Manolio 2013), while providing commentary on these matters for applied research on interventions for AMD. Key factors influencing the interpretation and application of genetic findings for clinical use include: (1) variant frequency and effect magnitude (based partially on genotyping feature probe sets); (2) the nature of variation (based on genotyping feature probe sets); and (3) alternative explanations for findings (based on coinherited variants with capacity to alter gene activity or protein structure).

\section{Variant Frequency and Effect Magnitude}

There is an unmet need to assess the magnitude of disease-gene associations from variants with minor allele frequencies $<5 \%$. Both common 
and rare variants have yielded useful information with the capacity for improving clinical care in AMD. It is likely that sets of common variants may act in aggregate to produce small but meaningful functional alterations in some people with AMD. The basis for this idea in complex disease etiology has been discussed in detail since the mid-1990s (Lander 1996; Manolio et al. 2009). Ward and Kellis (2012) have commented on works demonstrating the influence of common alleles contributing small but meaningful effects in traits or disease risk (Purcell et al. 2009); these loci, associated with complex traits at $P$-values far below those considered significant on a genome-wide level, have clustered across the genome within DNA sequence encoding constituents of biological pathways implicated in disease pathogenesis (Yang et al. 2010). A central premise is that inference based on a single locus may not elucidate important contributions of genetic variants both manifesting weak associations and the capacity to act together in alteration of disease risk (Lee et al. 2012; SanGiovanni and Lee 2013; Smoller et al. 2013). Robust findings have consistently emerged from the common variant approach to investigation, and such results have been successfully applied to identify systems acting in disease pathogenesis or pathophysiology (Manolio et al. 2009). As mentioned previously, the small proportion of trait heritability contributed by these common variants make them less than optimal for AMD risk prediction or classification (Jakobsdottir et al. 2009).

Disease-associated rare-frequency (minor allele frequency $<0.5 \%$ ) and low-frequency variants (minor allele frequency $0.5 \%-5.0 \%$ ) tend to have higher predictive value, and yield point estimates of higher magnitude than the common SNPs contained in most GWA gene chip probe feature sets. Stable estimates of effect (associations) for rare- and low-frequency variants require larger cohorts than those necessary for testing common variants. Efforts to identify rare variants have yielded promising results in AMD genetics (Raychaudhuri et al. 2011; Helgason et al. 2013; Seddon et al. 2013; Zhan et al. 2013). In some instances, high-impact rare causative variants may be resident in genes in which no common variants exist. If genes harboring common loci (likely detected with the GWA approach) are not proximal to those carrying rare variants, the process of detection is unlikely to be efficient. In this case, a pathway analysis may be useful to identify biologically plausible constituents for follow-up. Regions proximal to and within the 19 susceptibility loci reported by the AMD Genetics Consortium (Fritsche et al. 2013) are being evaluated with fine mapping and exome sequencing methods to expand knowledge on rare- and low-frequency variants associated with a doubling or more of AMD risk. Use of such variants in prediction models is expected to improve accuracy beyond levels explained by clinical, demographic, and lifestyle characteristics.

\section{The Nature of Variation}

A more sophisticated understanding of the structural nature of variation and nontranscribed DNA is a necessary condition for efficient translation of meaningful disease-variant findings that may not otherwise indicate obvious functional consequences. This process involves assessing the influence of genomic rearrangements and noncoding SNPs for their capacity to disrupt nontranscribed DNA involved in regulation of transcription. Structural variants and sites overlapping regulatory elements (transcription factor binding sites, DNase hypersensitivity clusters, microRNA-binding domains, and histone promoter or enhancer regions) are potentially important genomic elements in AMD susceptibility for which there is a lack of information. Many ( $>80 \%)$ GWA-defined risk loci fall within noncoding regions of the genome- as these regions are enriched with transcription enhancer elements, it is important to conduct large-scale projects to determine capacity of variation in noncoding and intergenic sequence to induce functional change (e.g., nucleotide changes that alter processing or expression of the gene transcript or structural and functional alterations in integrity of the protein). The ENCODE Project is now archiving such annotations (see https://www.genome .gov/encode/) in a public access database. 
Linkage Structure and Coinherited Variants of Functional Significance

We must thoroughly assess disease-SNP relationships for alternative explanations based on coinherited DNA variants-especially those with the capacity to influence gene transcription and protein structure. Disease-SNP relationships often are strongest for variants that have no apparent functional link to the encoded protein. Nonrandom cosegregation (coinheritance of DNA tracts in the genome) is one explanation for such observations-suggesting that the strongly associated SNP marks a susceptibility locus for a causative variant. Gains in accuracy of imputation (supported by the 1000 Genomes Project) and enhancements of annotation databases (supported by the ENCODE Project) have permitted an amplification of GWA study-derived signals with which to identify biologically meaningful findings. The application of whole-exome sequencing within loci identified through projects such as the AMD Gene Consortium (Fritsche et al. 2013) will target candidate functional variants.

The three factors discussed above are inextricably linked, and under certain circumstances may be used to: (1) increase discriminatory power for clinical risk assessment (through the application of predictive models); (2) distinguish clinical cohorts most likely to benefit from specific clinical interventions (through the application biomarkers with clinical information); and (3) assess genetic capacity for drug action and toxicity (through the applications of treatment response studies). We present overviews on these factors below.

\section{Risk Prediction}

AMD is a disease of public health significance, and early detection in the presence of an effective preventive intervention would alleviate a great burden of suffering. Most predictive models examining genetics-based causality of AMD are derived from measures of disease status (i.e., AMD prevalence). Gorin presents an astute commentary regarding the application of AMD genetics for risk prediction (Gorin 2012), stating that clinical applications leading to the most valuable preventive treatments should be informed by prospective studies assessing the genetic component of AMD incidence in people at moderate risk for developing advanced AMD. He notes that prevalent AMD can be predicted well in people carrying many or no susceptibility variants for the disease, but that existing models still lack sensitivity and specificity for the majority of people in the general population (most of whom carry a few risk variants). This point was initially elucidated by Jakobsdottir et al. (2009), who demonstrated that although accurate detection of $80 \%$ of AMD cases was possible with three strongly associated loci, prediction was poor for people without the disease (false-positive rates exceeded $40 \%$ at a sensitivity of $80 \%$ ). The second key point is that, until an effective preventive intervention for advanced AMD is developed, risk prediction will offer only limited value in clinical care. AMD is a common disease in the elderly, and costs associated with universal screening are not likely to be supported. With the advent of effective and accessible treatments, positive predictive values may be increased through a screening process focused on people with family histories of AMD and high genetic risk prediction scores. A number of clinical characteristics and lifestyle practices have been identified with AMD risk, and these may be combined with genetic risk scores from common and rare SNPs and structural variants to more accurately predict likelihood of AMD in people who would otherwise show low risks based on demographic or behavioral factors alone.

\section{Disease Classification and Subtyping}

Outside of the AMD field, disease-gene relationships and molecular subtyping of disease phenotypes have led to enhanced discrimination of subpopulations likely to benefit from specific treatments (Manolio 2013). This molecular subtyping has elucidated biomarkers that may best be applied in conditions in which other prognostic indicators are less sensitive and more difficult or expensive to obtain. The value of biomarkers is highest for people in preclinical 
disease phases and for those at moderate risk for progressing to advanced stages of complex disease (Manolio and Green 2011). AMD may manifest as a number of phenotypes (see $A M D$ Phenotypes section) that may not be accurately diagnosed in a clinical examination. The major clinical subtypes of advanced AMD (geographic atrophy and neovascular AMD) carry different risk factors and respond differently to a number of exposures (e.g., smoking) and therapies.

There is currently insufficient evidence to support the idea that the existing panel of serum biomarkers for AMD (C-reactive protein, complement component factors, VEGF, apo-lipoprotein $\mathrm{B}$, antiretinal antibodies, sterol ester levels, anticarboxyethylpyrrole adducts and autoantibodies, and vitamin D) contains effective predictors of AMD incidence or progression (Gorin 2012). Biomarkers have been proposed to support assessment of intervention efficacy, and there is currently an unmet need to demonstrate that long-term alteration of a biomarker by a controlled preventive or therapeutic intervention for AMD will avert subsequent development of AMD. Gorin emphasizes the importance of determining whether a biomarker is constrained to represent the current state of disease (static marker) or whether it may reflect a dynamic response of causal processes to an intervention.

\section{Genetic Basis of Drug Activity and Toxicity}

Interventions Based on AMD-Associated Pathway Constituents. Findings from the field of AMD genetics have been integrated with those from biochemical assessments of pathologic specimens in people with moderate or advanced AMD to guide research on the etiologic role of the complement system. Troutbeck et al. (2012) present a background on the complement pathways and their influence on proteolytic cascades that produce the cytolytic membrane attack complex. Complement component inhibitors, antibody-based compounds, and receptor antagonists have been examined as targets for AMD interventions (Table 1). For neovascular AMD, molecular targets within the VEGF system have been proposed and tested on the basis of biochemical analyses and the existence of related angiogenic and neovascular lesions in numerous blinding retinal diseases. The VEGFA gene resides in a susceptibility locus on chromosome 6, and aggregates of genes encoding constituents of the extracellular matrix (altered in vascular remodeling) have supported the decision to target therapies at VEGF (Table 1 contains a list of drugs inhibiting the VEGF system). Details on AMD-VEGF findings from clinical trials are reviewed by Miller (2013).

Drug Development and Drug Safety. Disease-gene relationships have been aimed at identifying novel molecular targets and determining whether DNA sequence variants influence an individual's ability to (1) activate prodrugs and drug-related metabolic or catabolic enzymes, and (2) respond to therapy for the purposes adjusting dosage or switching to alternative agents of higher efficacy. Gorin (2012) has reviewed the AMD pharmacogenomic literature and concluded that there is currently little evidence to support the claim that any DNA sequence variant alters response to treatment with VEGF inhibitors or photodynamic therapy. He further remarks that relationships of susceptibility loci have been additive in nature (each contributing an exclusive aspect of risk); when identified, molecular targets are likely to be heterogeneous and best treated with combined therapies with pharmacologic and nonpharmacologic approaches. Gorin also stresses the point that accurate quantitation of AMD phenotype is paramount in classifying disease progression for drug response studies. A recently published position statement is expected to offer guidance on this matter (Ferris et al. 2013). Finally, regarding constraints to progress in AMD pharmacogenomics, there currently are no established biomarkers with which to monitor response to preventive interventions in AMD-free people at moderate risk for developing the disease. For studies on progression to neovascular AMD, in vivo imaging technologies have been used successfully to identify biomarkers effective for assessing response to anti-VEGF therapies.

The Comparison of AMD Treatments Trials research team recently reported their inves- 
J.P. SanGiovanni and E.Y. Chew

Table 1. Therapeutic agents evaluated for AMD treatment

\begin{tabular}{|c|c|c|}
\hline Therapeutic agent & Target & Administration/Comment \\
\hline \multicolumn{3}{|l|}{ Complement System } \\
\hline \multicolumn{3}{|l|}{ Terminal Components } \\
\hline POT-4 & $\mathrm{C} 3$ & IVT/Inhibitory cyclic peptide \\
\hline ARC1905 & $\mathrm{C} 5$ & IVT/Inhibitory anti-C5 aptamer \\
\hline JSM-7717 & C5a receptor & Antagonistic receptor peptidomimetic \\
\hline JPE-1375 & C5a receptor & Antagonistic receptor peptidomimetic \\
\hline Eculizumab & $\mathrm{C} 5$ & $\mathrm{IV} / \mathrm{MAb}$ \\
\hline CR2-fH & $\mathrm{C} 3 \mathrm{~d} / \mathrm{CFH}$ & Recombinant $\mathrm{CFH}$, animal models \\
\hline \multicolumn{3}{|l|}{ Activation Components } \\
\hline FCFD4514S & CFD & IVT/Inhibitory MAb \\
\hline TA106 & $\mathrm{CFB}$ & INH/Inhibitory MAb \\
\hline \multicolumn{3}{|l|}{ VEGF System } \\
\hline Pegaptanib (Macugen) & Heparin domain VEGF- $A_{165}$ & $\begin{array}{l}\text { IVT/Inhibitory anti-VEGF } 165 \\
\text { aptamer }\end{array}$ \\
\hline Ranibizumab (Lucentis) & All VEGF-A isomers & IVT/Inhibitory MAb fragment \\
\hline Bevacizumab (Avastin) & All VEGF-A isomers & IVT/Inhibitory MAb \\
\hline Aflibercept (VEGF Trap) & All VEGF isomers, PIGF & $\begin{array}{l}\text { IVT/Fusion protein VEGFR1 + } \\
\text { VEGFR2 }\end{array}$ \\
\hline Pazopanib & VEGF Receptors, PDGFR & Topical/tyrosine kinase inhibitor \\
\hline AAV-sFLT1 & VEGFR & Recombinant VEGFR \\
\hline E10030 & VEGF, PDGF & anti-PDGF aptamer, used w/Lucentis \\
\hline RetinoStat & Angiostatin, Endostatin & Lentivirus vector \\
\hline ISONEP & Sphingosine-1-Phosphate & $\mathrm{MAb}$ \\
\hline Sirolimus (Rapamycin) & mTOR/Akt & IVT/Inhibitory macrolide \\
\hline OT-551 & $\mathrm{NF}-\kappa \mathrm{B}$ constituents & Topical \\
\hline AAV-PEDF & PEDF & IVT/SR, Recombinant PEDF, models \\
\hline
\end{tabular}

$\mathrm{AAV}$, adeno-associated virus; $\mathrm{C} 3$, complement component 3; C5, complement component 5; CFB, complement factor B; CFD, complement factor D; CFH, complement factor H; INH, inhaled; IVT, intravitreal injection; IV, intravenous injection; Mab, monoclonal antibody; mTOR, mammalian target of rapamycin; PDGF, platelet-derived growth factor; PDGFR, PDGF receptor; PEDF, pigment epithelium-derived growth factor; PlGF, placental growth factor; SR, subretinal; VEGF, vascular endothelial growth factor; VEGFR, VEGF receptor.

tigations on common AMD-associated SNPs (rs1061170 [CFH], rs10490924 [ARMS2], rs11200638 [HTRA1], and rs2230199 [C3]) and response to treatment with ranibizumab or bevacizumab for neovascular AMD (Hagstrom 2013). In no instance did the tested SNPs predict response to the study regimen of anti-VEGF therapy at one year. Endpoints included mean visual acuity (VA), mean change in VA, 15-letter or more increase in VA, retinal thickness, mean change in total foveal thickness, presence of fluid on ocular coherence tomography (OCT), presence of leakage on fluorescein angiography (FA), mean change in lesion size, and mean number of injections administered.

\section{CONCLUDING REMARKS}

We have provided an overview on the state of science regarding the potential clinical value of genetic investigations for improving AMD-related risk prediction, disease classification, and the genetic basis of drug activity and toxicity. Research teams are now expanding and improving assessment of rare, structural, and noncoding DNAvariants for the purposes of identifying genetic factors that might explain substantial proportions of variation in AMD outcomes. These findings will strengthen processes of prediction and classification. In 2013, an AMD phenotyping consortium published classification schemes that may be applied to assess 
changes in AMD associated with preventive and therapeutic interventions. Progress in developing effective clinical applications is dependent on successful integration of evidence from fields of molecular biology, biochemistry, biophysics, cell biology, and bioinformatics. A major constraint on risk prediction is the lack of information with which to characterize a genetics-based causality for AMD incidence and progression. Molecular subtyping of AMD phenotypes requires a set of dynamic biomarkers presenting prognostic value; although these have yet to be identified, the formation of multidisciplinary teams and their participation in largescale consortia offers promise for a more efficient biomarker characterization. A number of drugs targeting complement and VEGF systems are under evaluation, and forthcoming work on the role of noncoding DNA in AMD pathogenesis will likely lead to the identification of biochemical pathways enriched with genes containing AMD-associated sequence variants. Pharmacologic targets in these pathways may inform a rational and effective therapeutic approach to preventing and treating this sightthreatening disease. In conclusion, while the evidence from studies of AMD genetics is now best suited for informing preclinical research, it is a useful resource that may be effectively applied to improving clinical care.

\section{REFERENCES}

Ambati J, Ambati BK, Yoo SH, Ianchulev S, Adamis AP. 2003. Age-related macular degeneration: Etiology, pathogenesis, and therapeutic strategies. Surv Ophthalmol 48: 257-293.

Bindewald A, Schmitz-Valckenberg S, Jorzik JJ, DolarSzczasny J, Sieber H, Keilhauer C, Weinberger AW, Dithmar S, Pauleikhoff D, Mansmann U, et al. 2005. Classification of abnormal fundus autofluorescence patterns in the junctional zone of geographic atrophy in patients with age related macular degeneration. Br J Ophthalmol 89: $874-878$.

Bird AC. 2010. Therapeutic targets in age-related macular disease. J Clin Invest 120: 3033-3041.

Cichon S, Craddock N, Daly M, Faraone SV, Gejman PV, Kelsoe J, Lehner T, Levinson DF, Moran A, Sklar P, et al. 2009. Genomewide association studies: History, rationale, and prospects for psychiatric disorders. Am J Psychiatry 166: 540-556.

Ferris FL III, Wilkinson CP, Bird A, Chakravarthy U, Chew E, Csaky K, Sadda SR. 2013. Clinical classification of age- related macular degeneration. Ophthalmology 120: 844851.

Fritsche LG, Chen W, Schu M, Yaspan BL, Yu Y, Thorleifsson G, Zack DJ, Arakawa S, Cipriani V, Ripke S, et al. 2013 Seven new loci associated with age-related macular degeneration. Nat Genet 45: 433-439.

Gorin MB. 2012. Genetic insights into age-related macular degeneration: Controversies addressing risk, causality, and therapeutics. Mol Aspects Med 33: 467-486.

Haddad S, Chen CA, Santangelo SL, Seddon JM. 2006. The genetics of age-related macular degeneration: A review of progress to date. Surv Ophthalmol 51: 316-363.

Hageman GS, Gehrs K, Johnson LV, Anderson D. 2008. Agerelated Macular Degeneration (AMD). In Webvision: The Organization of the Retina and Visual System (ed. Kolb H, Nelson R, Fernandez E, Jones B) (source: http://webvision.med.utah.edu/)

Hagstrom SA, Ying G-S, Pauer GJ, Sturgill-Short GM Huang J, Callanan DG, Kim IK, Klein ML, Maguire MG, Martin DF. 2013. Pharmacogenetics for genes associated with age-related macular degeneration in the comparison of AMD treatments. Ophthalmology 120: 593-599.

Helgason H, Sulem P, Duvvari MR, Luo H, Thorleifsson G, Stefansson H, Jonsdottir I, Masson G, Gudbjartsson DF, Walters GB, et al. 2013. A rare nonsynonymous sequence variant in $C 3$ is associated with high risk of age-related macular degeneration. Nat Genet 45: 1371-1374.

Jakobsdottir J, Gorin MB, Conley YP, Ferrell RE, Weeks DE. 2009. Interpretation of genetic association studies: Markers with replicated highly significant odds ratios may be poor classifiers. PLoS Genet 5: e1000337.

Klaver CC, Wolfs RC, Assink JJ, van Duijn CM, Hofman A, de Jong PT. 1998. Genetic risk of age-related maculopathy. Population-based familial aggregation study. Arch Ophthalmol 116: 1646-1651.

Klein RJ, Zeiss C, Chew EY, Tsai JY, Sackler RS, Haynes C, Henning AK, SanGiovanni JP, Mane SM, Mayne ST, et al. 2005. Complement factor $\mathrm{H}$ polymorphism in age-related macular degeneration. Science 308: 385-389.

Lander ES. 1996. The new genomics: Global views of biology. Science 274: 536-539.

Lee PH, O’Dushlaine C, Thomas B, Purcell SM. 2012. INRICH: Interval-based enrichment analysis for genomewide association studies. Bioinformatics 28: 1797-1799.

Lim LS, Mitchell P, Seddon JM, Holz FG, Wong TY. 2012. Age-related macular degeneration. Lancet 379: 17281738.

Manolio TA. 2013. Bringing genome-wide association findings into clinical use. Nat Rev Genet 14: 549-558.

Manolio TA, Green ED. 2011. Genomics reaches the clinic: From basic discoveries to clinical impact. Cell 147: 1416.

Manolio TA, Collins FS, Cox NJ, Goldstein DB, Hindorff LA, Hunter DJ, McCarthy MI, Ramos EM, Cardon LR, Chakravarti A, et al. 2009. Finding the missing heritability of complex diseases. Nature 461: 747-753.

Manolio TA, Chisholm RL, Ozenberger B, Roden DM, Williams MS, Wilson R, Bick D, Bottinger EP, Brilliant MH, Eng C, et al. 2013. Implementing genomic medicine in the clinic: The future is here. Genet Med 15: 258-267. 
J.P. SanGiovanni and E.Y. Chew

McCarthy MI, Abecasis GR, Cardon LR, Goldstein DB, Little J, Ioannidis JP, Hirschhorn JN. 2008. Genome-wide association studies for complex traits: Consensus, uncertainty and challenges. Nat Rev Genet 9: 356-369.

Miller JW. 2013. Age-related macular degeneration revisited-piecing the puzzle: The LXIX Edward Jackson memorial lecture. Am J Ophthalmol 155: 1-35, e13.

Newman AM, Gallo NB, Hancox LS, Miller NJ, Radeke CM, Maloney MA, Cooper JB, Hageman GS, Anderson DH, Johnson LV, et al. 2012. Systems-level analysis of age-related macular degeneration reveals global biomarkers and phenotype-specific functional networks. Genome Med 4: 16.

Purcell SM, Wray NR, Stone JL, Visscher PM, O'Donovan MC, Sullivan PF, Sklar P. 2009. Common polygenic variation contributes to risk of schizophrenia and bipolar disorder. Nature 460: 748-752.

Raychaudhuri S, Iartchouk O, Chin K, Tan PL, Tai AK, Ripke S, Gowrisankar S, Vemuri S, Montgomery K, Yu $\mathrm{Y}$, et al. 2011. A rare penetrant mutation in $\mathrm{CFH}$ confers high risk of age-related macular degeneration. Nat Genet 43: $1232-1236$.

SanGiovanni JP, Lee PH. 2013. AMD-associated genes encoding stress-activated MAPK pathway constituents are identified by interval-based enrichment analysis. PLoS ONE 8: e71239.

SanGiovanni JP, Mehta S. 2009. Variation in lipid-associated genes as they relate to risk of advanced age-related macular degeneration. World Rev Nutr Diet 99: 105-158.

Seddon JM, Cote J, Page WF, Aggen SH, Neale MC. 2005 The US twin study of age-related macular degeneration: Relative roles of genetic and environmental influences. Arch Ophthalmol 123: 321-327.
Seddon JM, Yu Y, Miller EC, Reynolds R, Tan PL, Gowrisankar S, Goldstein JI, Triebwasser M, Anderson HE, Zerbib J, et al. 2013. Rare variants in CFI, C3 and C9 are associated with high risk of advanced age-related macular degeneration. Nat Genet 45: 1366-1370.

Silveira AC, Morrison MA, Ji F, Xu H, Reinecke JB, Adams SM, Arneberg TM, Janssian M, Lee JE, Yuan Y, et al. 2010. Convergence of linkage, gene expression and association data demonstrates the influence of the RAR-related orphan receptor $\alpha$ (RORA) gene on neovascular AMD: A systems biology based approach. Vision Res 50: 698-715.

Smoller JW, Craddock N, Kendler K, Lee PH, Neale BM, Nurnberger JI, Ripke S, Santangelo S, Sullivan PF. 2013. Identification of risk loci with shared effects on five major psychiatric disorders: A genome-wide analysis. Lancet 381: $1371-1379$.

Troutbeck R, Al-Qureshi S, Guymer RH. 2012. Therapeutic targeting of the complement system in age-related macular degeneration: A review. Clin Experiment Ophthalmol 40: $18-26$.

Ward LD, Kellis M. 2012. Interpreting noncoding genetic variation in complex traits and human disease. Nat Biotechnol 30: 1095-1106.

Yang J, Benyamin B, McEvoy BP, Gordon S, Henders AK, Nyholt DR, Madden PA, Heath AC, Martin NG, Montgomery GW, et al. 2010. Common SNPs explain a large proportion of the heritability for human height. $\mathrm{Nat} \mathrm{Ge}$ net 42: 565-569.

Zhan X, Larson DE, Wang C, Koboldt DC, Sergeev YV, Fulton RS, Fulton LL, Fronick CC, Branham KE, BraggGresham J, et al. 2013. Identification of a rare coding variant in complement 3 associated with age-related macular degeneration. Nat Genet 45: 1375-1379. 


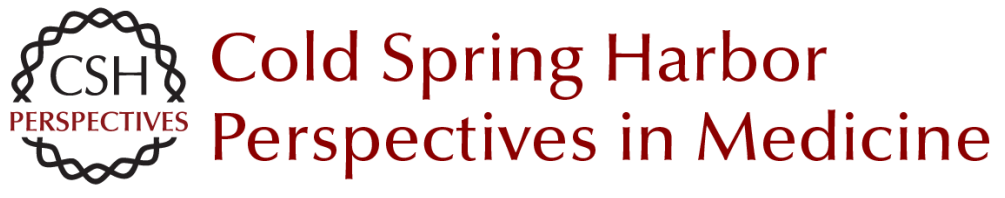

\section{Clinical Applications of Age-Related Macular Degeneration Genetics}

John Paul SanGiovanni and Emily Y. Chew

Cold Spring Harb Perspect Med 2014; doi: 10.1101/cshperspect.a017228 originally published online August 14, 2014

\section{Subject Collection Retinal Disorders: Genetic Approaches to Diagnosis and Treatment}

Trial by "Firsts": Clinical Trial Design and Regulatory Considerations in the Development and Approval of the First AAV Gene Therapy Product in the United States

Kathleen Z. Reape and Katherine A. High

Immunology of Retinitis Pigmentosa and Gene Therapy-Associated Uveitis

Paul Yang, Debarshi Mustafi and Kathryn L. Pepple

Developing New Vectors for Retinal Gene Therapy

Emilia A. Zin, Bilge E. Ozturk, Deniz Dalkara, et al.

Beyond the NEI-VFQ: Recent Experience in the Development and Utilization of Patient-Reported Outcomes for Inherited Retinal Diseases

Todd Durham, Judit Banhazi, Francesco Patalano, et al.

Electronic Retinal Prostheses

Daniel Palanker

Alternative RNA Splicing in the Retina: Insights and Perspectives

Casey J. Keuthan, Sadik Karma and Donald J. Zack

X-Linked Retinoschisis

Cristy A. Ku, Lisa W. Wei and Paul A. Sieving

A Systematic Review of Optogenetic Vision Restoration: History, Challenges, and New Inventions from Bench to Bedside

Antonia Stefanov and John G. Flannery
Lessons Learned from the Development of the

First FDA-Approved Gene Therapy Drug,

Voretigene Neparvovec-rzyl Jean Bennett and Albert M. Maguire

Therapeutic Gene Editing in Inherited Retinal Disorders Jinjie Ling, Laura A. Jenny, Ashley Zhou, et al.

Cell-Based Therapies: Strategies for Regeneration Marina Pavlou and Thomas A. Reh

The Importance of Natural History Studies in Inherited Retinal Diseases

Allison Ayala, Janet Cheetham, Todd Durham, et al.

Photoreceptor Cell Replacement Using

Pluripotent Stem Cells: Current Knowledge and Remaining Questions

Christelle Monville, Olivier Goureau and Karim Ben M'Barek

iPSC-RPE in Retinal Degeneration: Recent

Advancements and Future Perspectives

Tadao Maeda and Masayo Takahashi

Retinal Degeneration Animal Models in Bardet-

Biedl Syndrome and Related Ciliopathies Clarisse Delvallée and Hélène Dollfus

Mobility Testing and Other Performance-Based

Assessments of Functional Vision in Patients with Inherited Retinal Disease

Daniel Chung, Colas Authié and Laure Blouin

For additional articles in this collection, see http://perspectivesinmedicine.cshlp.org/cgi/collection/ 
For additional articles in this collection, see http://perspectivesinmedicine.cshlp.org/cgi/collection/ 